\title{
Ontology-based Tamil-English cross-lingual information retrieval system
}

\author{
D THENMOZHI* and CHANDRABOSE ARAVINDAN
}

Department of Computer Science and Engineering, SSN College of Engineering, Kalavakkam 603 110, India e-mail: theni_d@ssn.edu.in; aravindanc@ssn.edu.in

MS received 15 November 2014; revised 6 July 2017; accepted 24 June 2018; published online 14 August 2018

\begin{abstract}
Cross-lingual information retrieval (CLIR) systems facilitate users to query for information in one language and retrieve relevant documents in another language. In general, CLIR systems translate query in source language to target language and retrieve documents in target language based on the keywords present in the translated query. However, the presence of ambiguity in source and translated queries reduces the performance of the system. Ontology can be used to address this problem. The current approaches to ontology-based CLIR systems use manually constructed multilingual ontology, which is expensive. However, many methods exist to automatically construct ontology for any domain in English but not in other languages like Tamil. We propose a methodology for Tamil-English CLIR system by translating the Tamil query to English and retrieve pages in English to address these issues. Our approach uses a word sense disambiguation module to resolve the ambiguity in Tamil query. An automatically constructed ontology in English is used to address the ambiguity of English query. We have developed a morphological analyser for Tamil language, Tamil-English bilingual dictionary and named entity database to translate a Tamil query to English. The translated query is reformulated using ontology and the reformulated queries are given to a search engine to retrieve English documents from the Internet. We have evaluated our methodology for agriculture domain and the evaluation results show that our approach outperforms other approaches in terms of precision.
\end{abstract}

Keywords. Cross-lingual information retrieval system; ontology; Tamil-English query translation; query expansion; semantic web.

\section{Introduction}

Internet provides a rich source of information and is growing at an enormous rate. English is still the dominant language in the Internet, which contributes most of the information. However, world Internet usage statistics reveal that the number of non-English Internet users is steadily increasing, but all of them are not able to formulate queries in English. Tamil users such as farmers and people working for small scale industries who are not able to express their needs in English are also growing in the Internet. They generally search for information using Tamil search engines. But the content provided by these search engines is not adequate. Making the huge repository of information on the Web, which is available in English, accessible to nonEnglish Internet users has become an essential challenge in recent times. When the non-English users want to access the existing search engines, most of the time they formulate improper English queries.

Cross-lingual information retrieval (CLIR) systems aim to solve the afore-mentioned problem by allowing the users

*For correspondence to express their information need in their native language while the CLIR system takes care of matching it appropriately with the relevant documents in the target language. In general, CLIR systems translate the query in source language to target language and retrieve documents in target language. When the translated query has multiple meanings, all the documents that are retrieved may not be relevant to the user. For example, the user query "payinkaal" is translated to "tiller", which has multiple meanings, namely part of a boat, agriculture equipment and name of a person. All the retrieved documents are not relevant to the user. Hence, it is necessary to include semantics into the search process to retrieve only relevant pages to the users. Also, the search process is improved by refining the queries to more specific queries. It is difficult for the Tamil users who are not able to express their needs in English to formulate such refined queries. We propose an ontology-based CLIR system that suggests possible refined queries and retrieves documents for all the queries.

Many research works have been reported for handling semantics in information retrieval (IR) using ontology [1-5]. Queries are accepted in formal languages like SPARQL in these research works. It is difficult for the users 
such as farmers to pose such queries. CLIR systems [6-10] facilitate non-English users to pose natural language queries in their own languages but fail to handle semantics. A few research works [11-15] have been reported on ontology-based CLIR systems that deal with semantics using bilingual ontologies. However, very few approaches are evolved to build multilingual ontologies [16-18] automatically from available resources like text documents, databases, etc. Also, many methods exist to automatically construct ontology for any domain in English but not in other languages. No such methodologies exist for learning Tamil ontology. Hence, we use a word sense disambiguation (WSD) module to resolve the ambiguity in Tamil queries during translation.

We propose a CLIR system in agricultural domain for Tamil farmers. The system retrieves relevant documents from an English corpus in response to a query expressed in Tamil language. Here, the query given in Tamil language is translated syntactically and semantically to English for IR process. The ambiguity of the translated query can be resolved by reformulating the query using an ontology. The ambiguity still persists even if we use a general purpose ontology like WordNet. For example, when we use WordNet, the query "Tiller" is reformulated as "Tiller Shoot", "Tiller Farmer", "Tiller Lever", "Tiller is part of Rudder", "Harrow Tiller", and "Tiller Farm Machine". Among these queries, "Tiller Shoot", "Tiller Lever" and "Tiller is part of Rudder" will not retrieve any pages related to agriculture equipment. Hence, it is important to use a domain-specific ontology to reformulate the queries. We use an agriculture ontology that has been learnt from text documents automatically [19].

Section 2 briefly describes various works related to ontology-based retrieval and CLIR systems. Section 3 elaborates our framework designed for cross-lingual semantic retrieval system. Section 4 provides the details of experiments conducted to analyse the performance of the proposed ontology-based CLIR system. Section 5 gives conclusion and future directions for this research.

\section{Related work}

IR is the process of extracting relevant information for the given query. The huge increase in the amount of information in the Internet and the complexity to reach such information caused an excessive demand for tools and techniques that can handle data semantically [2]. Ontology-based retrieval is a solution to semantic web. However, many ontology-based retrieval systems do not deal with cross-language issues. Several approaches are reported to address the cross-language issues but fail to deal with ambiguity problems. A few research methodologies have been reported that deal with both cross-language and semantic issues but have many open issues. This section reviews existing research works and open issues related to ontology-based retrieval, CLIR and ontology-based CLIR.

\subsection{Ontology-based retrieval}

Bhogal et al [20] and Jain and Singh [21] presented a comprehensive survey on query expansion using ontology for IR. Zimmermann et al [1] extended RDF framework and SPARQL language by annotating with more information for representing, reasoning and querying semantic web data. Kara et al [2] proposed a methodology for semantic retrieval based on domain ontology. They proved that the methodology outperforms traditional keyword-based methods and query expansion methods. However, the queries are extended based only on the class hierarchy information of the ontology, but not based on the semantic relationships of the ontology. Also, the method retrieves information only from a set of documents that are semantically indexed.

Mustafa et al [3] proposed an approach to ontologybased semantic IR. The query in triple form is matched with a triple in ontology and gets reformulated with the ontology terms for retrieval. They have evaluated 300 manually collected documents in the domain of research thesis. The approach does not handle incomplete and imprecise triples of the queries. Also, the approach can be extended for cross-lingual applications. Hogan et al [4] implemented a semantic web search engine that consists of components of IR system such as crawling, data enhancing, indexing and a user interface for search, browsing and retrieval of information. This search engine operates on RDF framework of ontology.

Fernandez et al [5] introduced an ontology-based approach for semantically enhanced IR. In this approach, the query is accepted in a formal SPARQL language, lists of semantic entities are returned and documents that are indexed with these semantic entities are retrieved. This IR system requires the user to be familiar with the formal languages like SPARQL. It is desirable to have a common IR system that can be used by any user who does not have formal language knowledge. Sy et al [22] developed a usercentred and ontology-based IR system in which the given query is reformulated either by adding or removing concepts from the query. This is done by graphically selecting the documents as interested or not interested by the user. This IR is semi-automatic due to query refinement using explicit specification of interest.

\subsection{CLIR}

Sujatha and Dhavachelvan [23] presented a survey on CLIR and multilingual information retrieval (MLIR) systems in Indian and Foreign languages. Sorg and Cimiano [6] developed a CLIR system using cross-language links of Wikipedia. The user can give query in English, French and 
German languages and retrieve documents from English corpus or from German corpus. They developed a model to map bag of words that represent a document to bag of concepts using Wikipedia. They [24] extended this approach by analysing different strategies for exploiting the Wikipedia structure to define the concept space. Evaluations have been performed for both CLIR and MLIR systems for English, French, German and Spanish languages. However, ambiguity of the query in source and translated languages is not resolved in these approaches.

Several organizations in India are working on the CLIR system for Indian Languages [25]. Bandyopadhyay et al [8] developed a Bengali, Hindi and Telugu-English CLIR system as part of the ad-hoc bilingual task. Chinnakotla et al [9] developed Hindi-English and Marathi-English CLIR systems. Pingali and Varma [10] developed a Hindi and Telugu-English CLIR system. Mandal et al [26] developed a CLIR system for two most widely spoken Indian languages, Hindi and Bengali. All these works use bilingual dictionaries. Jagarlamudi and Kumaran [27] also worked on Hindi-English cross-lingual system in which a word alignment table was used that was learnt by a statistical machine translation (MT) system trained on aligned parallel sentences. All these research methodologies have been evaluated for English corpus of LA Times 2002. Rao and Devi [28] developed Tamil-English CLIR Track for news articles taken from "The Telegraph", English news magazine in India. All these approaches use word by word translation method in news domain.

Sivakumar et al [7] developed a Hindi-English CLIR system that identifies equivalent English document for the given Hindi document based on cosine similarity measure. The features of the documents to find the similarity are reduced using latent semantic indexing. This approach requires a parallel corpus that contains documents in both languages. This system works well for document queries but not for user-generated queries.

Thenmozhi and Aravindan [29] developed a CLIR for Tamil farmers using MT approach. This approach translates the Tamil query to English using a morphological analyser, bi-lingual dictionary and NE recognizer. WSD is incorporated to avoid ambiguity in Tamil to English translation. However, the methodology does not handle the ambiguity in the translated query.

\subsection{Ontology-based CLIR}

Yu et al [11] developed a Chinese-English CLIR system based on domain ontology. Abusalah et al [13] developed an Arabic-English CLIR system based on ontology for travel and tourism. Yahya et al [12] developed EnglishMalay and Malay-English CLIR systems based on Quran ontology. However, the methodologies require ontologies in both source and target languages. Construction of multilingual ontology is a time-consuming task. Ontologies are built manually in these research works. Methodologies for constructing such ontologies automatically from existing resources like text document, databases, etc. are not available. Also, the approaches do not consider the semantic relationships of the ontology to improve the retrieval performance.

Monti et al [14] proposed a methodology for ontologybased CLIR. Italian-English retrieval has been evaluated using this approach for archaeological domain. This approach uses ontology for source language to refine the query and then translated to the target language. However, ambiguity in the translated query is not resolved by this approach, which may occur frequently especially in English language. Pourmahmoud and Shamsfard [15] developed a Persian-English CLIR system using ontology. Bilingual ontology and dictionary are used to translate the query to the target language query. Ontology is used to disambiguate the meaning of source query to target query when the source query has multiple meanings in target query. Probabilistic approach has been used to disambiguate the target query in this research. However, suggesting more refined queries to the user is not supported by this retrieval system.

By considering several issues discussed in this section, we propose a framework for CLIR system that addresses the ambiguity in both source language and target languages to improve the retrieval performance.

\section{Proposed methodology}

The proposed Tamil-English CLIR system translates the given Tamil query to an English query and also suggests multiple reformulated queries for searching and retrieval using ontology. This process is depicted in figure 1 .

\subsection{Morphological analysis}

The words present in the given query are transformed to their root form using a morphological analyser that uses several rules for handling plurals, case suffixes, oblique,

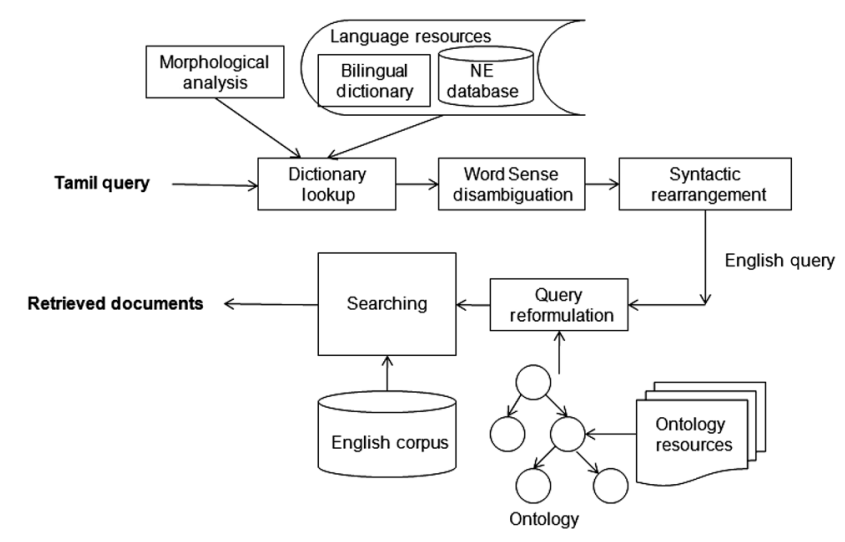

Figure 1. System architecture. 
etc. The morphological analyser identifies the root form of the word and its suffixes. In Tamil, "kaL" is the major plural suffix. It has variants like "tkaL", "NGkaL", "RkaL" and "KkaL". After removing the suffix "kaL", the morphological analyser modifies the root word to get its base form by replacing "NG with $\mathrm{m}$ ", "R with 1 ", etc. Postpositions, namely accusative, dative, genitive, locative and plain postpositions, come next to case suffices like ai, in, il, itam, etc. The morphological analyser removes these postpositions along with case suffixes to bring the word to its root form. A list of different types of postpositions is given as follows:

- Accusative postpositions: vita, pola, kontu, nokki, patti, kuRittu, cuRRi, vittu, thavira, munnittu, venti, otti, poRuttu, poRuttavari

- Dative postpositions: aaka, enRu, mun, pin, ul, itaiye, natuve, mattiyil, veliye, mel, kizh, etiril, pakkattil, arukil, patil, maaraaka, neRaaka, uriya, ulla, takunta

- Genetive postpositions: mitu, mel, valiyaaka, mUlamaaka, vazhiyaaka, pEril, poRuttu

- Locative postpositions: irunthu, occurs only after case markers itam and il

- Plain postpositions: utan, kUta, utaiya, vacam, itam, varai, aaka, toRum, aara

- Oblique suffix: ththu.

Table 1 shows some of the compound words in Tamil and their root words along with suffixes. This analyser identifies multiples of suffixes to convert the word to its root form. For example, root word "maram" is obtained from the word "marangkaLinvazhiyaaka" (through trees) by removing multiple suffixes.

Table 1. Examples for morphological analysis.

\begin{tabular}{|c|c|c|c|}
\hline Word & Suffix & Suffix type & $\begin{array}{l}\text { Root } \\
\text { word }\end{array}$ \\
\hline puukkaL (flowers) & kkaL & plural & puu \\
\hline marangkaL (trees) & ngkaL & plural & maram \\
\hline naatkaL (days) & tkaL & plural & naaL \\
\hline kaRkaL (stones) & RkaL & plural & kal \\
\hline avanai vita (than him) & ai-vita & $\begin{array}{l}\text { Accusative } \\
\text { postposition }\end{array}$ & avan \\
\hline avanukkenRu (for him) & ukk-enRu & $\begin{array}{l}\text { Dative } \\
\text { postposition }\end{array}$ & avan \\
\hline $\begin{array}{l}\text { kathavinmel (on the } \\
\text { door) }\end{array}$ & In-mel & $\begin{array}{l}\text { Genitive } \\
\text { postposition }\end{array}$ & kathavu \\
\hline $\begin{array}{l}\text { avanitamirutnthu(from } \\
\text { him) }\end{array}$ & $\begin{array}{l}\text { Itam- } \\
\text { irutnthu }\end{array}$ & $\begin{array}{l}\text { Locative } \\
\text { postposition }\end{array}$ & avan \\
\hline $\begin{array}{l}\text { viidu varai (to the } \\
\text { house) }\end{array}$ & varai & $\begin{array}{c}\text { Plain } \\
\text { postposition }\end{array}$ & viidu \\
\hline patikka (to study) & kka & $\begin{array}{c}\text { Non-finite form } \\
\text { of verb }\end{array}$ & pati \\
\hline maraththu (tree) & ththu & Oblique & maram \\
\hline
\end{tabular}

\subsection{Dictionary look-up}

We have used a bi-lingual dictionary to obtain the English translation of the Tamil words. The dictionary look-up process uses the morphological analyser and sandhi rules to obtain the English translation of the Tamil words. The steps involved in obtaining the English translation of the query are given in Algorithm 1. The algorithm accepts a sequence of Tamil words $T$ as input and gives a sequence of English words $E$. Each word in $T$ is first searched in a named entity (NE) database to obtain its transliteration. If $T$ is not present in the NE database, the word is searched in the dictionary to obtain its English translation. If the word is not present in the dictionary, we remove the suffix of the query term and obtain its root word using the morphological analyser. Then the root word is searched in NE database and in bilingual dictionary for its transliteration and translation, respectively. For example, for the query "ponni arisi" (ponni rice), the word "ponni" is present in NE database and is transliterated. The word "arisi" is translated as "rice" using the dictionary. However, some parts of named entities need to be translated. For example, for the query, "Maduraiyil paayum nathikaL" (rivers flow in Madurai), the term "Maduraiyil" is searched in NE database and there is no such entry in it. Then the term is searched in the dictionary and it is not found in the dictionary too. Next, the morphological analyser identifies the root term "Madurai" and its suffix "yil" for the word. Then these lexical units are searched in the NE database and in the dictionary. The term "Madurai" is transliterated using the NE database and the suffix "yil" is translated to "in" using the dictionary. If the term is not present in both NE database and dictionary after removal of all suffixes, then it is added to the target query as it is. If the word of the query has multiple meanings from the dictionary, we use WSD to obtain a single meaning. For example, for the query, "manjal valarkka ettra mann" (soil suitable for growing turmeric), the word "manjaL" has two meanings in the dictionary namely "turmeric" and "yellow". We obtain the meaning as "turmeric" using Algorithm 2. Algorithm 1 finds the sequence of set of translations $E_{s}$ for all the words present in $T$. For example, for the query, "manjaL vaLarkka ettra mann", $E_{s}=<\{-$ turmeric, yellow $\}$, \{grow $\}$, \{suitable for $\},\{$ soil $\}>$. This algorithm returns the sequence of English translations as $E=<$ turmeric, grow, suitable for, soil $>$ using Algorithm 2 .

If the root form of the word is not directly present in the dictionary, we use sandhi rules, namely, "U removal", "VY adding", "Doubling", "TR replacement" and "KCH-TH-P" rules to split the word into two. For example, the query word "nerpayir" (paddy crop) can be transformed to "nel" "payir" using the "TR replacement" rule before obtaining the translation. If the root form of the word cannot be split, then this dictionary look-up process removes each suffix of the root form of the word until there is an entry in the dictionary to find the translation. For example, for the given word "veeLaanmai" (agriculture), 
meaning agriculture, the root word available in the dictionary is "veeLaan". Removing the suffix "mai", the translation for "veeLaan" is extracted as "agriculture".

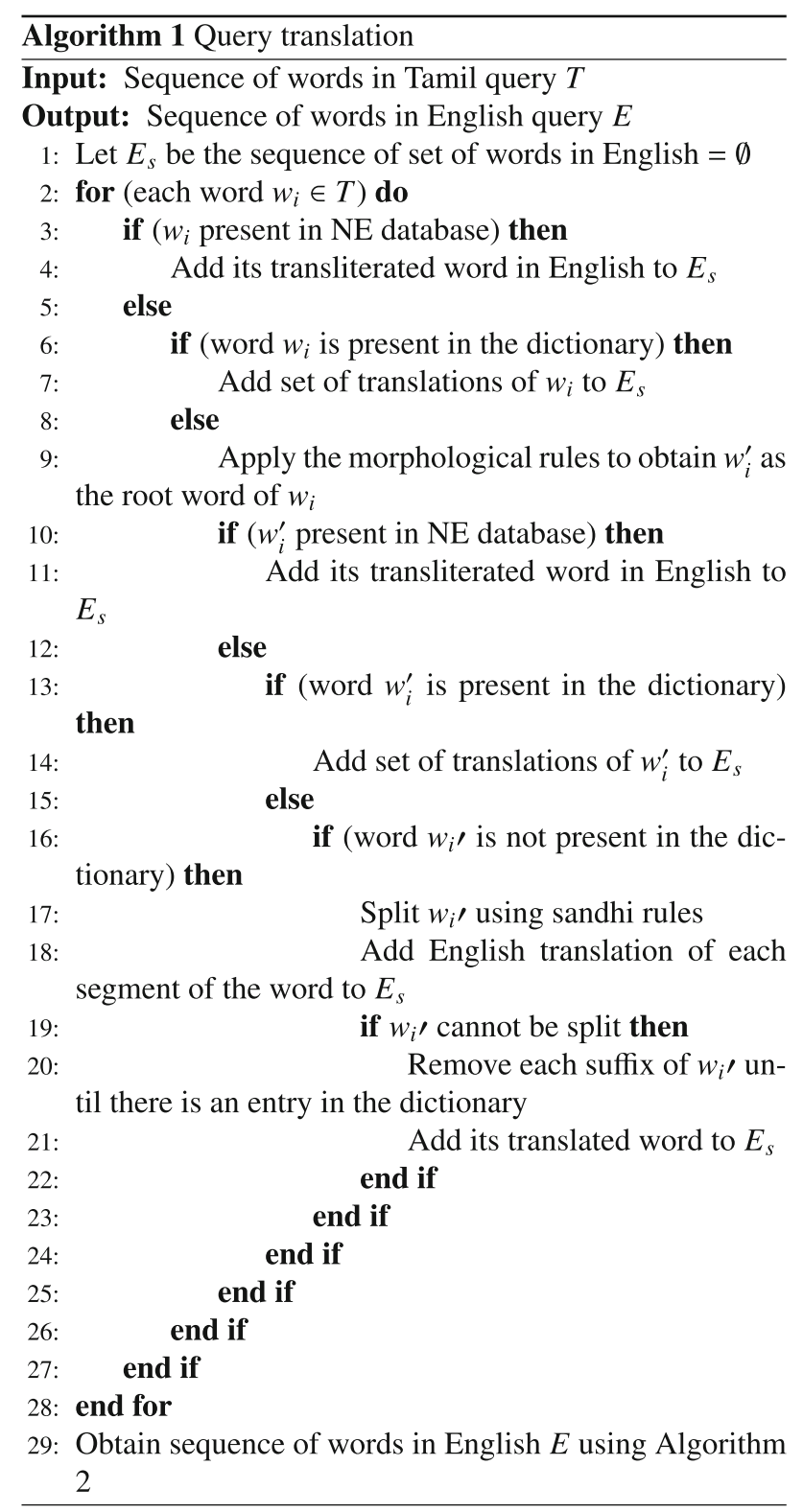

Table 2 shows some of the examples for obtaining meaning using dictionary look-up. Since the dictionary is built from the scratch as no resource is available for this domain, the system exhibits a dynamic learning approach wherein any new word that is encountered in the translation process may be added to the bilingual dictionary by allowing the user to dynamically insert into the dictionary along with its corresponding English meaning.

\subsection{WSD}

The process for WSD is presented in Algorithm 2. When a word in the query has multiple senses, then for each sense
Table 2. Examples for query translation.

\begin{tabular}{|c|c|c|}
\hline Query & $\begin{array}{l}\text { Meaning from } \\
\text { dictionary }\end{array}$ & $\begin{array}{l}\text { Steps } \\
\text { used }\end{array}$ \\
\hline veeLaanmai (agriculture) & agriculture & $20-21$ \\
\hline pooni arisi (ponni rice) & ponni rice & $3-4,6-7$ \\
\hline $\begin{array}{l}\text { veeLaanmai katan thittangkal } \\
\text { (agriculture loan plans) }\end{array}$ & $\begin{array}{l}\text { agriculture loan } \\
\text { plan }\end{array}$ & $13-14$ \\
\hline nerpayir (paddy crop) & paddy crop & 16-18 \\
\hline manjaL vaLarkka ettra mann & $\begin{array}{c}\{\text { turmeric,yellow }\} \\
\text { grow }\end{array}$ & $6-7$ \\
\hline $\begin{array}{l}\text { (soil suitable for growing } \\
\text { turmeric) }\end{array}$ & suitable for soil & \\
\hline veeLan (agriculture) & agriculture & $6-7$ \\
\hline $\begin{array}{l}\text { Maduraiyil paayum nathikaL } \\
\text { (rivers flow in Madurai) }\end{array}$ & $\begin{array}{l}\text { in Madurai flow } \\
\text { rivers }\end{array}$ & $\begin{array}{r}10-11 \\
13-14\end{array}$ \\
\hline
\end{tabular}

of a given word, it is compared to all possible senses of the surrounding words in the given query. The count of number of words common between the sense descriptions is calculated and assigned as the score for the particular sense of the word. The sense that has the highest score is declared the most appropriate one for the target word in the given context. For example, the query "manjaL vaLarkka ettra mann" has ambiguous meaning for the word "manjaL". It has two different translations namely "yellow" and "turmeric". To disambiguate this, WordNet sense information is obtained for "yellow" and "turmeric". After removing all the stop words, the key terms are retrieved. Similarly, key terms for the surrounding words namely "soil" and "grow" are obtained from the WordNet sense information. Surrounding words are obtained by removing the word with ambiguous meaning and the stop words. The key terms for the word with different senses and the surrounding words are listed in table 3.

The process for WSD is presented in Algorithm 2. It accepts the sequence of set of English translations $E_{s}$ and gives the sequence of English translations $E$. For example, the algorithm accepts $E_{s}=<\{$ turmeric, yellow $\},\{$ grow $\}$, \{suitable for $\},\{$ soil $\}>$ as input. Surrounding words are obtained by extracting the sets of $E_{S}$ with cardinality 1 and removing the words in the sets if they are stop words. Thus the surrounding words are "grow" and "soil". Find the surrounding words sense set $K_{s}$ by extracting all the words except stop words from the sense information of the words, namely grow and soil. The set $e_{i}$ in $E_{s}$ with a cardinality greater than one is considered to be a word with multiple meanings. For each word $e_{j}$ in $e_{i}$, extract all the words except stop words from the sense information as word set $K_{e j}$ and count the number of common elements $v_{e j}$ between $K_{e j}$ and $K_{s}$. For this example, we have obtained $V_{\text {turmeric }}=1$ and $v_{\text {yellow }}=0$. The term with maximum sense score is considered as a single term after eliminating ambiguity. Thus "turmeric" is added as an element to $E$. Finally, this algorithm returns the sequence of English translations as $E=<$ turmeric, grow, suitable for, soil $>$. 
Table 3. Keyterms from WordNet senses for query terms.

\begin{tabular}{lcccc}
\hline WordNet senses & & & \multicolumn{2}{c}{ Surrounding words } \\
\cline { 1 - 2 } \cline { 5 - 5 } Turmeric & Yellow & & Soil & Grow \\
\hline cultivate & yellow & & plant & corn \\
tropical & color & & grow & grow \\
plant & pigment & & earth & forest \\
India & chromatic & & land & mushroom \\
yellow & resembling & & plow & tree \\
fower & hue & & agriculture & hair \\
aromatic & sunflower & & soil & vegetable \\
rhizome & ripe & & & backyard \\
source & lomon & & \\
condiment & & & \\
dye & & & \\
\hline
\end{tabular}

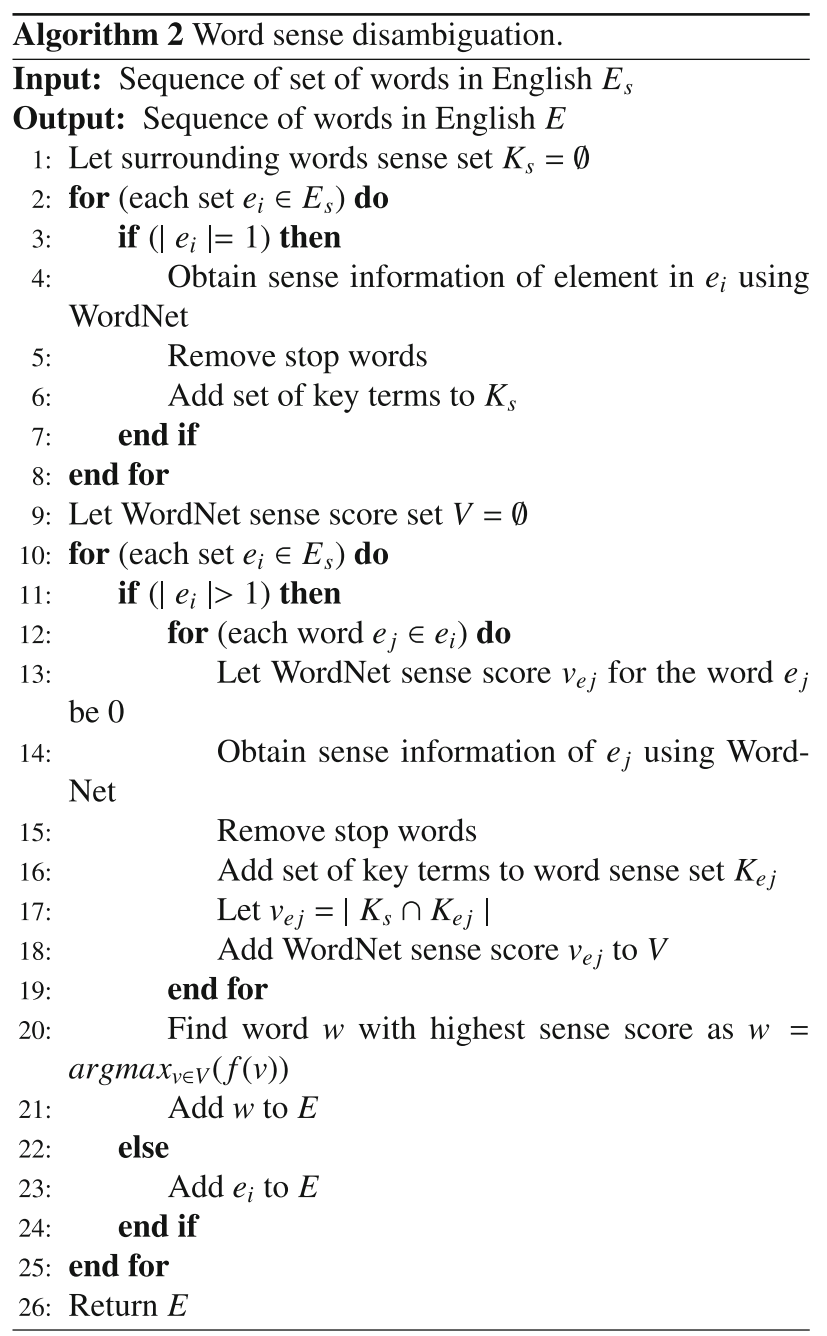

\subsection{Syntactic rearrangement}

CLIR focuses on the cross-language issues from the IR perspective rather than MT perspective [10]. However, syntactic rearrangement (SR) of the translated queries may give better search results. Also, it gives more clarity to the user about the translated query. For example, a Tamil query "udal nalaththirrku ettra payirkaL" (crops suitable for body health) is translated to English query "body health suitable for crops" in a word by word approach. The search engines retrieve "body health" related pages to the top. If the query is rearranged to "crops suitable for body health", it may give a better clarity and search result. Tamil is a subject-object-verb (SOV) language in which the sentence is present in subject, object and verb order. However, English is primarily a subject-verb-object (SVO) language. Tamil-English query translation involves identifying the individual translated words into subject, verb and object and placing them in correct order. In order to perform the translation, part of speech (POS) information is added for all the words in the dictionary. A local word reordering is performed based on POS tagging to obtain SVO pattern of English query [30].

\subsection{Query reformulation}

The translated query may be ambiguous. When the terms of the translated query (English) have ambiguous meaning, most of the pages of the search result would be irrelevant with respect to agriculture domain. It is apparent that refining the query to more specific query will improve the performance of the search result. For example, the Tamil query "mutkalappai" (harrow) is translated to English query "harrow" using this approach. When this query is given to a search engine like Google, most of the pages are not relevant to agriculture equipment. The query term "harrow" has several meanings such as area in London, school, software, actress and harrow council along with agriculture equipment. It is necessary to resolve this ambiguity. Also, refining query to more specific query in English is difficult for Tamil users. Hence, it is important to help the user with possible queries related to the given query. This refinement may be possible with the help of general purpose ontology like WordNet. However, this ontology neither refines the query to eliminate the ambiguity (i.e., it refines to more specific queries in all domains) nor performs any refinement. For example, when we use WordNet, the translated query "Plough" is reformulated as "Plough is part of Great Bear", "Asterism Plough", "Bull Tongue Plough", "Mouldboard Plough Plough" and "Plough Tool". Among these queries, only the last two queries are related to agriculture equipment. Also, WordNet does not give any related terms for the queries like "Traction Equipment". Hence, it is important to use a domain-specific ontology to reformulate the queries. We use agriculture ontology that has been learnt automatically from text documents [19] to reformulate the query and to suggest with possible refined queries in agriculture domain. A part of domain-specific ontology is shown in figure 2 . The query reformulation (QR) using ontology is also useful 


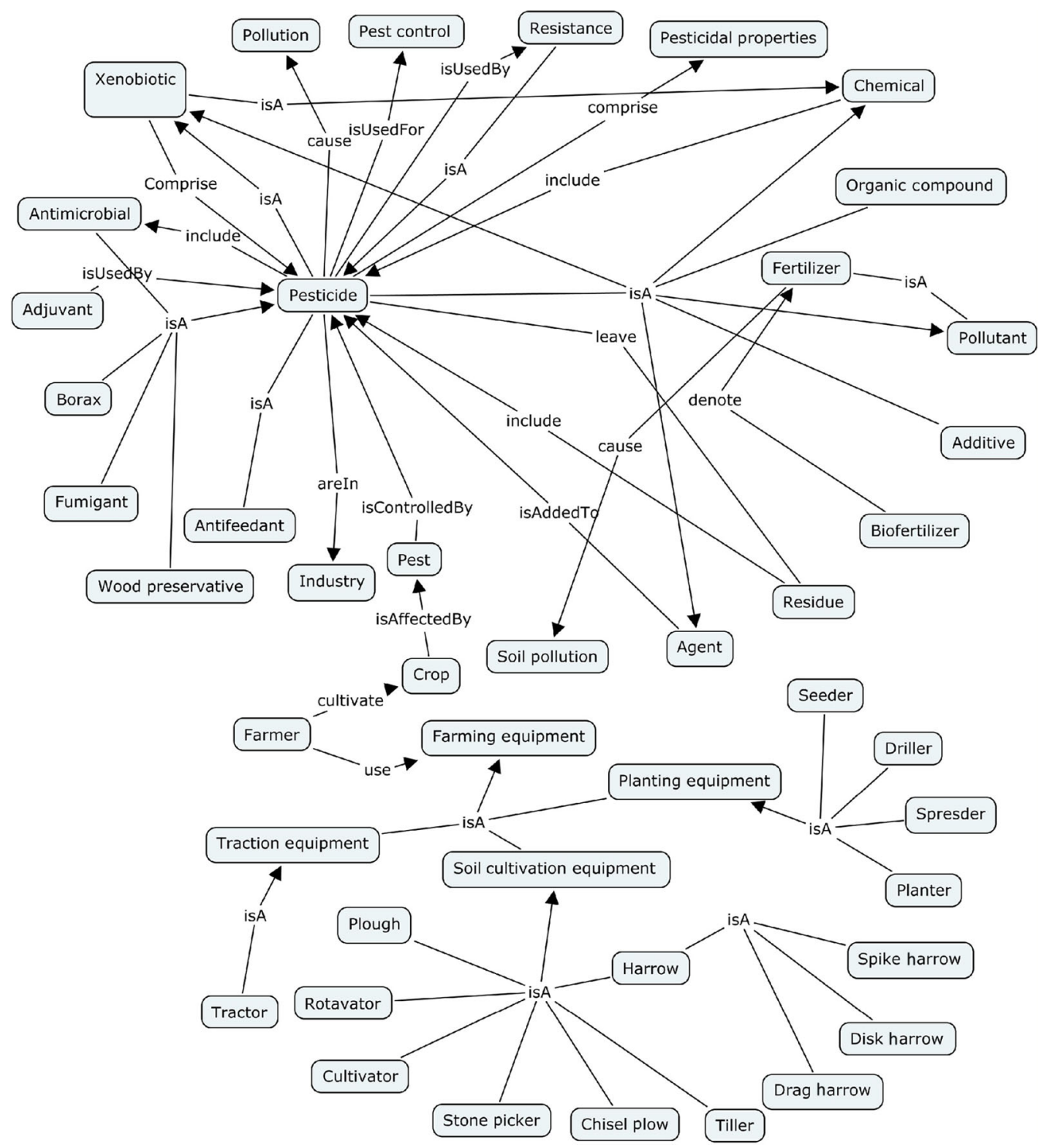

Figure 2. Agriculture ontology.

to disambiguate the source query that cannot be handled by our WSD approach. For example, let us consider the query "ManjaLin payankaL" (uses of turmeric). The term "ManjaL" has two meanings namely "yellow" and "turmeric" in the dictionary. Since, the sense of the surrounding word "payankaL" does not contribute to resolve this ambiguity, our WSD will not be helpful. In this case, both the translations, namely "uses of yellow" and "uses of turmeric", will be given to QR process where the term "turmeric" is present in the ontology. Thus the query "uses of turmeric" will be retained by refining it as "uses of turmeric + crop", and the other query is ignored for the search process.

Ontology is represented as a digraph $A=\langle C, R\rangle$, where $C=\left\{c_{1}, \ldots c_{m}\right\}$ and $R=\left\{r_{1} \ldots r_{n}\right\}$.

- Let $q$ be the translated query string.
- Let $S$ be the set of reformulated queries.

- For any concept $\left(c_{i} \in C\right)=q$, extract all $c_{j}$, if $c_{j}$ is an adjacent node of $c_{i}$.

- Generate reformulated query set $S$ for $q$ by adding elements using a function for all $c_{j}$.

$$
f\left(c_{i}, c_{j}\right)=\left\{\begin{array}{cc}
1 . c_{j}+c_{i}, & \text { if }\left(c_{i}, c_{j}\right) \mapsto r \text { and } \\
r=\text { hierarchical relation } & \\
2 . c_{i}+r+c_{j}, & \text { if }\left(c_{i}, c_{j}\right) \mapsto r \text { and } \\
r \neq \text { hierarchical relation } & \\
3 . c_{i}+c_{j}, & \text { if }\left(c_{j}, c_{i}\right) \mapsto r \text { and } \\
r=\text { hierarchical relation } & \\
4 . c_{j}+r+c_{i}, & \text { if }\left(c_{j}, c_{i}\right) \mapsto r \text { and } \\
r \neq \text { hierarchical relation } &
\end{array}\right.
$$

For example, let translated query $q=$ "harrow". Elements of reformulated query set $S$ are 
- "harrow + soil cultivation equipment" by Function 3

- "disk harrow + harrow" by Function 1

- "drag harrow + harrow" by Function 1

- "spike harrow + harrow" by Function 1

For the translated query $q=$ "pest", elements of $S$ are

- "pest is control by pesticide" by Function 2

- "crop is affect by pest" by Function 4

\subsection{Searching}

The reformulated queries are converted into URLs for the search engine that is being used. The URLs are then passed on to the browser which retrieves the relevant documents from the Internet and the search results are displayed to the user.

\subsection{Walk through examples}

We consider two examples to show the significance of all the processes involved in our approach. However, all the processes are not useful for all the queries. The examples are given here.

1. Vaigai aatril uLLa miin vagaikaL (fish types present in Vaigai river).

2. ManjaLin payankaL (uses of turmeric).

\section{Query 1: Vaigai aatril uLLa miin vagaikaL}

Steps involved:

1. Tokenize the query into terms.

2. The term "Vaigai" is searched first in NE database.

3. It is found and it is transliterated. Now the resultant query term is $\{$ Vaigai $\}$.

4. The term "aatril" is searched in NE database.

5. It is not found, hence the morphological analyser identifies the root word as "aaru" using Sandhi rule from "aatr" and the suffix "il".

6. Then the word "aaru" is searched in NE database.

7. It is not found and hence "aaru" is searched in TamilEnglish dictionary.

8. Two English translations are obtained for "aaru" from the dictionary and thus we get the resultant query terms $<$ Vaigai, $\{$ in river, in six $\}>$.

9. Next, the third term "uLLa" is searched in NE database. It is not found and hence searched in the dictionary and the translation "present" is obtained. Thus the resultant query terms are $<$ Vaigai, in river, in six $\}$, present $>$.

10. The fourth term "miin" is searched in NE database. It is not found and hence searched in dictionary and the translation "fish" is obtained, which results in the query terms $<$ Vaigai, $\{$ in river, in six $\}$, present, fish $>$.
11. The last term "vagaikaL" is searched in NE database. It is not found; then the suffix "kaL" is removed and the remaining word "vagai" is searched in NE database. It is not found and hence "vagai" is searched in the dictionary; there it is found as "type".

12. The translation for "kaL" is appended to "type" and thus the resultant query terms are $<$ Vaigai, $\{$ river, six $\}$, present in, fish, types $>$.

13. To perform WSD, the surrounding terms obtained after removing stop words are \{vaigai, fish, types considered for both the queries.

14. The WordNet sense information of these terms is compared to the sense information of "river" and "six". The sense score for "river" is higher than the sense score of "six", which results in the query terms $<$ Vaigai, in river, present, fish, types $>$.

15. The MT process transforms the positions of the "Vaigai river" and "fish types", resulting in the query "fish types present in Vaigai river".

16. Then each term is searched in agriculture ontology for further refinement. Currently, our agriculture ontology does not contain any of the concepts present in the query.

17. The QR is not performed for this target query and the final query is "fish types present in Vaigai river", which is used for searching process.

\section{Query 2: ManjaLin payankaL}

Steps involved:

1. The term "manjaLin" is searched in NE database. It is not found; hence the suffix "in" is removed and the remaining term "manjaL" is searched in NE database, which is not present there.

2. The term "manjaL" is now searched in the dictionary and there are two translations, namely "yellow" and "turmeric", found in the dictionary.

3. The resultant query terms are <of, \{yellow, turmeric $\}>$.

4. The second word "payankaL" is search in NE database. It is not found; hence the suffix "kaL" is removed and the remaining term "payan" is searched in NE database, which is not present there, and hence it is searched in the dictionary.

5. The translation for "payan" is obtained as "use" from the dictionary. By adding the translation of the suffix "kaL", we obtain the resultant term as "uses". Thus the resultant query terms are <of, \{yellow, turmeric\}, uses $>$.

6. The WSD process removes the stopword "of" and extracts the surrounding term as "uses".

7. The sense information of "uses" is compared to the senses of "yellow" and "turmeric".

8. There is no sense score obtained for both "yellow" and "turmeric", which result in two queries "of yellow uses" and "of turmeric uses". 
Table 4. Results of our approach for the queries.

\begin{tabular}{|c|c|c|c|}
\hline Query no. & Source query & Translated query & Precision (\%) Top 20 \\
\hline Q1 & $\begin{array}{c}\text { ManjaL vaLarkka ettra mann } \\
\text { (Soil suitable for growing turmeric) }\end{array}$ & $\begin{array}{l}\text { Soil suitable for } \\
\text { grow turmeric }\end{array}$ & 100 \\
\hline Q2 & $\begin{array}{l}\text { Vaigai aatril uLLa miin vagaikaL } \\
\text { (Fish types present in Vaigai river) }\end{array}$ & $\begin{array}{l}\text { Fish types present in } \\
\text { Vaigai river }\end{array}$ & 65 \\
\hline Q3 & $\begin{array}{l}\text { Maduraiyil paayum nathikaL } \\
\text { (Rivers flow in Madurai) }\end{array}$ & Rivers flow in Madurai & 95 \\
\hline Q4 & $\begin{array}{l}\text { Udal nalaththirrku ettra payirkaL } \\
\text { (Crops suitable for body health) }\end{array}$ & $\begin{array}{l}\text { Crops suitable for } \\
\text { body health }\end{array}$ & 95 \\
\hline Q5 & $\begin{array}{c}\text { ManjaL } \\
\text { (Turmeric) }\end{array}$ & Turmeric crop & 100 \\
\hline Q6 & $\begin{array}{l}\text { VeNkaaram } \\
\text { (Borax) }\end{array}$ & Borax pesticide & 95 \\
\hline Q7 & $\begin{array}{l}\text { EthiruutikaL } \\
\text { (Antifeedants) }\end{array}$ & Antifeedants pesticide & 85 \\
\hline Q8 & $\begin{array}{l}\text { Thunai marunthu poruL } \\
\text { (Adjuvants) }\end{array}$ & $\begin{array}{l}\text { Adjuvant is used } \\
\text { by pesticide }\end{array}$ & 100 \\
\hline Q9 & $\begin{array}{l}\text { ManjaLin payankaL } \\
\text { (Uses of turmeric) }\end{array}$ & Uses of turmeric crop & 100 \\
\hline Q10 & $\begin{array}{l}\text { Mutkalappai } \\
\text { (Harrow) }\end{array}$ & $\begin{array}{l}\text { 1. Harrow Soil Cultivation } \\
\text { Equipment } \\
\text { 2. Drag Harrow Harrow } \\
\text { 3. Disk Harrow Harrow } \\
\text { 4. Spike Harrow Harrow }\end{array}$ & 100 \\
\hline Q11 & $\begin{array}{l}\text { Uzhudhal UpakaranangkaL } \\
\text { (Traction Equipments) }\end{array}$ & $\begin{array}{l}\text { 1. Traction Equipment } \\
\text { Agriculture Equipment } \\
\text { 2. Tractor Traction } \\
\text { Equipment }\end{array}$ & 100 \\
\hline Q12 & $\begin{array}{l}\text { Payinkaal } \\
\text { (Tiller) }\end{array}$ & $\begin{array}{l}\text { 1. Tiller Soil } \\
\text { Cultivation Equipment } \\
\text { 2. Power Tiller Tiller } \\
\text { 3. Rotary Tiller Tiller }\end{array}$ & 100 \\
\hline Q13 & $\begin{array}{l}\text { PuussikaL } \\
\text { (Pests) }\end{array}$ & $\begin{array}{l}\text { 1. Crop affect Pest } \\
\text { 2. Pest control Pesticide }\end{array}$ & 100 \\
\hline Q14 & $\begin{array}{l}\text { Kalappai } \\
\text { (Plough) }\end{array}$ & $\begin{array}{c}\text { Plough Soil Cultivation } \\
\text { Equipment }\end{array}$ & 100 \\
\hline
\end{tabular}

9. The MT process transforms these queries to "uses of yellow" and "uses of turmeric".

10. The QR process refines the word "turmeric" to "turmeric crop" and thus the resultant queries are "uses of yellow" and "uses of turmeric crop".

\section{Implementation and experiments}

\subsection{Implementation}

We have evaluated the performance of ontology-based Tamil-English CLIR system in agriculture domain. Several queries formulated by Tamil farmers have been used to evaluate the performance of our system. The queries we have used for evaluation are of 5-6 words length. Hence, the context window for translation includes the complete query to determine for target query. We have developed a Tamil-English bilingual dictionary of size $6.08 \mathrm{MB}$ that contains most of the words related to agricultural domain. We have built an NE database with 3611 entities, including 2580 place names, 132 river and lake names, and 899 person names with respect to Tamilnadu. We have collected the data from Internet ${ }^{1,2,3,4}$ to build the NE database. We have used a rule-based morphological analyser developed for Tamil-English CLIR system [29]. This analyser is similar to Amritha's morphological analyser [31]. Our morphological analyser finds the root term of the query and its various suffixes by handling suffixes and sandhi rules. We use agriculture ontology, which has been automatically learnt from text [19] to resolve the ambiguity in the translated queries.

\footnotetext{
${ }^{1}$ http://www.fallingrain.com/world/IN/25/.

${ }^{2}$ https://en.wikipedia.org/wiki/List_of_rivers_of_Tamil_Nadu.

${ }^{3}$ https://en.wikipedia.org/wiki/List_of_lakes_in_Tamil_Nadu.

${ }^{4}$ https://en.wikipedia.org/wiki/List_of_Tamil_people.
} 
Table 5. Results of our experiments.

\begin{tabular}{|c|c|c|c|c|c|c|c|}
\hline \multirow[b]{2}{*}{ Query no. } & \multirow[b]{2}{*}{ Source query } & \multicolumn{6}{|c|}{ P@20 (\%) } \\
\hline & & E1 & E2 & E3 & E4 & E5 & E6 \\
\hline Q1 & ManjaL vaLarkka ettra mann & 0 & 100 & 100 & 0 & 100 & 100 \\
\hline Q2 & Vaigai aatril uLLa miin vagaikaL & 45 & 35 & 65 & 30 & 35 & 65 \\
\hline Q3 & Maduraiyil paayum nathikaL & 95 & 85 & 95 & 85 & 85 & 95 \\
\hline Q4 & Udal nalaththirrku ettra payirkaL & 95 & 90 & 95 & 90 & 90 & 95 \\
\hline Q5 & ManjaL & 100 & 100 & 50 & 100 & 50 & 100 \\
\hline Q6 & VeNkaaram & 95 & 95 & 25 & 95 & 25 & 95 \\
\hline Q7 & EthiruutikaL & 85 & 85 & 45 & 85 & 45 & 85 \\
\hline Q8 & Thunai marunthu poruL & 100 & 100 & 0 & 100 & 0 & 100 \\
\hline Q9 & ManjaLin payankaL & 100 & 100 & 50 & 100 & 50 & 100 \\
\hline Q10 & Mutkalappai & 100 & 100 & 0 & 100 & 0 & 100 \\
\hline Q11 & Uzhudhal upakaranangkaL & 100 & 100 & 0 & 100 & 0 & 100 \\
\hline Q12 & Payinkaal & 100 & 100 & 10 & 100 & 10 & 100 \\
\hline Q13 & PuussikaL & 100 & 100 & 45 & 100 & 45 & 100 \\
\hline \multirow[t]{2}{*}{ Q14 } & Kalappai & 100 & 100 & 10 & 100 & 10 & 100 \\
\hline & $\operatorname{MAP}(\%)$ & 86.79 & 92.14 & 42.14 & 84.64 & 38.93 & 95.36 \\
\hline
\end{tabular}

\subsection{Experiments}

The performance of any retrieval system can be analysed by the metrics precision and recall. We have not evaluated our methodology with a finite number of documents set as proposed in [12] and [15], wherein the list of relevant pages are known to measure recall. We have utilized full-text search engines like Google, which returns a huge number of pages for the queries, and hence the performance is measured in terms of only precision. Precision is calculated for top 20 pages (P@20) retrieved by the search engine that are mostly viewed by the users. Precision is measured by providing web-based user interface to the domain experts to mark the retrieved pages that are relevant to the query or not. The results of our approach for various queries are shown in table 4 . We have obtained a mean average precision of $95.36 \%$ for P@20.

We have performed the following six experiments to ascertain the significance of the components, namely WSD, $\mathrm{SR}$ and $\mathrm{QR}$, using ontologies that are employed in our approach.

E1: experiments without WSD

E2: experiments without SR

E3: experiments without $\mathrm{QR}$

E4: experiments without WSD and SR

E5: experiments without $\mathrm{SR}$ and $\mathrm{QR}$

E6: experiments with all components

The performance of all these six experiments in terms of P@20 is presented in table 5.

It is observed from table 5 that our ontology-based retrieval, which includes all the components, namely WSD, $\mathrm{SR}$ and $\mathrm{QR}$, where the translation quality is high, gives a mean average precision of $95.36 \%$ for P@20. The translation quality is reduced due to the absence of any components used in our approach. Table 5 shows that the experiments without ontology reduce the retrieval performance to $42.14 \%$ and $38.93 \%$. However, WSD and SR also contribute to the performance of the retrieval. The error rates for all the six experiments are $0.13,0.08,0.58,0.15$, 0.61 and 0.05 . This shows that the error rate is very much reduced when all the components, namely WSD, SR and $\mathrm{QR}$, are involved in the translation process (E6), where the translation quality is high. The SR has lesser impact in the retrieval performance (E2). However, the absence of ontology considerably increases the error rates to 0.58 (E3) and 0.61 (E5).

\subsection{Perforamance comparison of search methods}

It is evident from table 5 that ontology significantly improves the performance of the retrieval system. The ontology may be a general purpose ontology or a domain-

Table 6. Query types.

\begin{tabular}{lc}
\hline Query type & Meaning \\
\hline SGT & Source query to Google Tamil \\
SY & Source query to Yahoo \\
SWU & Source query to Web Ulagam \\
STW & Source query to Tamil Wikipedia \\
TG & Translated query by Google \\
TCLIR & Translated query by CLIR [29] \\
RCLIRGO & Reformulated query by CLIR using \\
RCLIRDO & General Purpose Ontology \\
& Reformulated query by CLIR using \\
\hline
\end{tabular}


Table 7. Performance comparison of search methods.

\begin{tabular}{lcccccccc}
\hline & \multicolumn{7}{c}{ P@ 20 $(\%)$} \\
\cline { 2 - 8 } Query no. & SGT & SY & SWU & STW & TG & TCLIR & RCLIRGO & RCLIRDO \\
\hline Q1 & 80 & 85 & 0 & 10 & 0 & 100 & 100 & 100 \\
Q2 & 50 & 30 & 0 & - & 65 & 65 & 65 & 65 \\
Q3 & 60 & 35 & - & 95 & 95 & 95 & 95 & 95 \\
Q4 & 70 & 100 & - & 0 & 20 & 95 & 30 & 95 \\
Q5 & 60 & 85 & 60 & 50 & 0 & 50 & 0 & 100 \\
Q6 & 15 & - & - & - & 25 & 25 & 15 & 95 \\
Q7 & - & 0 & - & - & - & 45 & 45 & 85 \\
Q8 & 15 & 0 & - & 5 & 0 & 0 & 0 & 100 \\
Q9 & 100 & 100 & 100 & 5 & 0 & 0 & 66.67 & 100 \\
Q10 & - & - & 0 & 0 & - & 100 & 100 & 100 \\
Q11 & 60 & 35 & 0 & 0 & 100 & 0 & 0 & 100 \\
Q12 & 0 & 0 & 0 & 0 & - & 10 & 48.33 & 100 \\
Q13 & 100 & 85 & 100 & 100 & 100 & 45 & 18.75 & 100 \\
Q14 & 40 & 5 & - & 25 & 7.5 & 5 & 33 & 100 \\
MAP & 46.43 & 40 & 18.57 & 20.71 & 29.46 & 45.36 & 44.05 & 95.36 \\
\hline
\end{tabular}

specific ontology. To ascertain the significance of domainspecific ontology in the retrieval performance, we have compared both variations (using general purpose and domain-specific ontology) of our ontology-based crosslingual retrieval performance to Tamil search engines, namely Google Tamil, Yahoo, Web Ulagam and Tamil Wikipedia that use keyword search, queries translated by Google for the given query and CLIR system proposed in [29], which translates the Tamil query to English using MT approach. Table 6 shows the different query types used for comparing the search methods. The P@20 values of different search methods are summarized in table 7 .

It is observed from table 7 that the performance of ontology-based CLIR using agriculture ontology is better than those of the other search methods. The results of individual queries for various search methods are given in Appendix I to show the significance of domain purpose ontology.

\section{Conclusions}

The proposed ontology-based CLIR system helps Tamil farmers to pose their query in Tamil and to retrieve documents from the Internet in English. The ambiguity in Tamil query is addressed using WSD. The ambiguity in the translated query is resolved using agriculture ontology, which has been learnt from text documents automatically. This CLIR system helps the user with more possible queries. These queries are semantically relevant to the given query, unlike Google, which suggest based on some keywords that are used to index the documents. We have evaluated our system by using several queries framed by Tamil farmers. We have measured the performance using the metric precision. We have performed different experiments to ascertain the importance of various components, namely WSD, $\mathrm{SR}$ and $\mathrm{QR}$, using ontologies that are employed in our approach. We have compared our ontology-based system to conventional keyword search using several Tamil search engines, CLIR system and Google translation system. Our system outperforms the other methods in terms of mean average precision. Our system retrieves the highly ranked pages to top 20, unlike other Tamil search engines. This system can be further extended to provide a summary in English for top pages, translate the summary to Tamil or provide an answer to the query in Tamil like an expert system.

\section{Acknowledgements}

We would like to thank the management of SSN Institutions for funding the High Performance Computing (HPC) lab, where this research is being carried out. We also thank the anonymous reviewers for their constructive comments, which helped us to improve the manuscript.

\section{Appendix I}

Tables $8,9,10,11,12,13,14,15$ and 16 show the significance of domain-specific ontology in the retrieval performance by comparing with other search methods. 
Table 8. Performance comparison for the user query "Mutkalappai".

\begin{tabular}{lccc}
\hline & & \multicolumn{2}{c}{ Precision $(\%)$} \\
\cline { 3 - 4 } & & Top & Top \\
Query & Query type & 10 & 20 \\
\hline Mutkalappai & SGT & 33.3 & - \\
Mutkalappai & SY & 20.0 & - \\
Mutkalappai & SWU & 0 & 0 \\
Mutkalappai & STW & 0 & 0 \\
Mutkalappai & TG & - & - \\
Harrow & TCLIR & 100 & 100 \\
Disk Harrow Harrow & RCLIRGO & 100 & 100 \\
Harrow Cultivator & RCLIRGO & 100 & 100 \\
Harrow Tiller & RCLIRGO & 100 & 100 \\
Harrow Soil Cultivation & RCLIRDO & 100 & 100 \\
$\quad$ Equipment & & & \\
Drag Harrow Harrow & RCLIRDO & 100 & 100 \\
Disk Harrow Harrow & RCLIRDO & 100 & 100 \\
Spike Harrow Harrow & RCLIRDO & 100 & 100 \\
\hline
\end{tabular}

Table 9. Performance comparison for the user query "Uzhudhal Upakaranangkal".

\begin{tabular}{lccc}
\hline & Query & \multicolumn{2}{c}{ Precision (\%) } \\
\cline { 3 - 4 } Query & type & Top 10 & Top 20 \\
\hline Uzhudhal Upakaranangkal & SGT & 70 & 60 \\
Uzhudhal Upakaranangkal & SY & 70 & 35 \\
Uzhudhal Upakaranangkal & SWU & 0 & 0 \\
Uzhudhal Upakaranangkal & STW & 0 & 0 \\
Tillage Equipment & TG & 100 & 100 \\
Traction Equipment & TCLIR & 0 & 0 \\
Traction Equipment & RCLIRGO & 0 & 0 \\
Traction Equipment Agriculture & RCLIRDO & 100 & 100 \\
Equipment & & & \\
Tractor Traction Equipment & RCLIRDO & 100 & 100 \\
\hline
\end{tabular}

Table 10. Performance comparison for the user query "Payinkaal".

\begin{tabular}{lccc}
\hline & Query & \multicolumn{2}{c}{ Precision $(\%)$} \\
\cline { 2 - 4 } Query & type & Top 10 & Top 20 \\
\hline Payinkaal & SGT & 0 & 0 \\
Payinkaal & SY & 0 & 0 \\
Payinkaal & SWU & 0 & 0 \\
Payinkaal & STW & 0 & 0 \\
Payinkaal & TG & - & - \\
Tiller & TCLIR & 10 & 10 \\
Tiller Shoot & RCLIRGO & 0 & 0 \\
Tiller Farmer & RCLIRGO & 100 & 95 \\
Tiller Lever & RCLIRGO & 0 & 0 \\
Tiller is part of Rudder & RCLIRGO & 0 & 0 \\
Harrow Tiller & RCLIRGO & 100 & 100 \\
Tiller Farm Machine & RCLIRGO & 90 & 95 \\
Tiller Soil Cultivation Equipment & RCLIRDO & 100 & 100 \\
Power Tiller Tiller & RCLIRDO & 100 & 100 \\
Rotary Tiller Tiller & RCLIRDO & 100 & 100 \\
\hline
\end{tabular}

Table 11. Performance comparison for the user query "Puussikal".

\begin{tabular}{lccc}
\hline & & \multicolumn{2}{c}{ Precision $(\%)$} \\
\cline { 2 - 4 } Query & type & Top 10 & Top 20 \\
\hline Puussikal & SGT & 100 & 100 \\
Puussikal & SY & 90 & 85 \\
Puussikal & SWU & 100 & 100 \\
Puussikal & STW & 100 & 100 \\
Insects & TG & 100 & 100 \\
Pest & TCLIR & 10 & 45 \\
Pest Epidemic Disease & RCLIRGO & 80 & 50 \\
Bubonic Plague Pest & RCLIRGO & 0 & 0 \\
Pneumonic Plague Pest & RCLIRGO & 0 & 0 \\
Septicemic Plague Pest & RCLIRGO & 0 & 0 \\
Nudnik Pest & RCLIRGO & 0 & 0 \\
Pest Tormentor & RCLIRGO & 0 & 15 \\
Vermin Pest & RCLIRGO & 80 & 85 \\
Pest Animal & RCLIRGO & 0 & 0 \\
Crops affect Pest & RCLIRDO & 100 & 100 \\
Pest control Pesticide & RCLIRDO & 100 & 100 \\
\hline
\end{tabular}

Table 12. Performance comparison for the user query "Kalappai".

\begin{tabular}{lccc}
\hline & & \multicolumn{2}{c}{ Precision $(\%)$} \\
\cline { 3 - 4 } Query & type & Top 10 & Top 20 \\
\hline Kalappai & SGT & 50 & 40 \\
Kalappai & SY & 0 & 5 \\
Kalappai & SWU & 10 & - \\
Kalappai & STW & 30 & 25 \\
Plow & TG & 10 & 10 \\
Plough & TG & 10 & 5 \\
Plough & TCLIR & 10 & 5 \\
Plough is part of Great Bear & RCLIRGO & 0 & 0 \\
Asterism Plough & RCLIRGO & 0 & 0 \\
Bull Tongue Plough & RCLIRGO & 0 & 0 \\
Mouldboard Plough Plough & RCLIRGO & 90 & 80 \\
Plough Tool & RCLIRGO & 70 & 85 \\
Plough Soil Cultivation & RCLIRDO & 100 & 100 \\
\multicolumn{1}{c}{ Equipment } & & & \\
\hline
\end{tabular}

Table 13. Performance comparison for the user query "VeNkaaram".

\begin{tabular}{lccc}
\hline & \multirow{2}{c}{$\begin{array}{c}\text { Query } \\
\text { type }\end{array}$} & Top 10 & Top 20 \\
\cline { 3 - 4 } Query & SGT & 20 & 15 \\
VeNkaaram & SY & - & - \\
VeNkaaram & SWU & - & - \\
VeNkaaram & STW & - & - \\
VeNkaaram & TG & 30 & 25 \\
Borax & TCLIR & 30 & 25 \\
Borax & RCLIRGO & 20 & 15 \\
Mineral borax & RCLIRDO & 100 & 95 \\
Borax pesticide & & &
\end{tabular}


Table 14. Performance comparison for the user query "EthiruutikaL".

\begin{tabular}{lccc}
\hline & \multirow{2}{c}{$\begin{array}{c}\text { Query } \\
\text { type }\end{array}$} & Top 10 & Top 20 \\
\cline { 3 - 4 } Query & SGT & - & - \\
\hline EthiruutikaL & SY & 0 & 0 \\
EthiruutikaL & SWU & - & - \\
EthiruutikaL & STW & - & - \\
EthiruutikaL & TG & - & - \\
EthiruutikaL & TCLIR & 50 & 45 \\
antifeedants & RCLIRGO & 50 & 45 \\
antifeedants & RCLIRDO & 90 & 85 \\
antifeedants pesticide & Ro &
\end{tabular}

Table 15. Performance comparison for the user query "Thunai Marunthu PoruL".

\begin{tabular}{lccc}
\hline & Query & \multicolumn{2}{c}{ Precision (\%) } \\
\cline { 3 - 4 } Query & type & Top 10 & Top 20 \\
\hline Thunai Marunthu PoruL & SGT & 20 & 15 \\
Thunai Marunthu PoruL & SY & 0 & 0 \\
Thunai Marunthu PoruL & SWU & - & - \\
Thunai Marunthu PoruL & STW & 10 & 5 \\
Adjuvant & TG & 0 & 0 \\
Adjuvant & TCLIR & 0 & 0 \\
Adjuvant & RCLIRGO & 0 & 0 \\
Adjuvant is used by pesticide & RCLIRDO & 100 & 100 \\
\hline
\end{tabular}

Table 16. Performance comparison for the user query "ManjaLin payankaL".

\begin{tabular}{lccc}
\hline & & \multicolumn{2}{c}{ Precision (\%) } \\
\cline { 2 - 4 } Query & type & Top 10 & Top 20 \\
\hline ManjaLin payankaL & SGT & 100 & 100 \\
ManjaLin payankaL & SY & 100 & 100 \\
ManjaLin payankaL & SWU & 100 & 100 \\
ManjaLin payankaL & STW & 10 & 5 \\
Uses of yellow & TG & 0 & 0 \\
Uses of yellow & TCLIR & 0 & 0 \\
Uses of yellow pigment & RCLIRGO & 0 & 0 \\
Uses of turmeric plant & RCLIRGO & 100 & 100 \\
Uses of turmeric food & RCLIRGO & 100 & 100 \\
Uses of turmeric crop & RCLIRDO & 100 & 100 \\
\hline
\end{tabular}

\section{References}

[1] Zimmermann A, Lopes N, Polleres A and Straccia U 2012 A general framework for representing, reasoning and querying with annotated semantic web data. Web Semant. Sci. Serv. Agents World Wide Web 11: 72-95

[2] Kara S, Alan Ö, Sabuncu O, Akpınar S, Cicekli N K and Alpaslan F N 2012 An ontology based retrieval system using semantic indexing. Inf. Syst. 37(4): 294-305

[3] Mustafa J, Khan S and Latif K 2008 Ontology based semantic information retrieval. In: Proceedings of the 4th
International IEEE Conference on Intelligent Systems, IS'08, vol. 3, pp. 2214-2219

[4] Hogan A, Harth A, Umbrich J, Kinsella S, Polleres A and Decker S 2011 Searching and browsing linked data with SWSE: the semantic web search engine. Web Semant. Sci. Serv. Agents World Wide Web 9(4): 365-401

[5] Fernández M, Cantador I, López V, Vallet D, Castells P and Motta E 2011 Semantically enhanced information retrieval: an ontology-based approach. Web Semant. Sci. Serv. Agents World Wide Web 9(4): 434-452

[6] Sorg P and Cimiano P 2008 Cross-lingual information retrieval with explicit semantic analysis. In: Working Notes for the CLEF 2008 Workshop

[7] SivaKumar A P, Premchand P and Govardhan A 2011 Indian languages IR using latent semantic indexing. Int. J. Comput. Sci. Inf. Technol. 3: 245-253

[8] Bandyopadhyay S, Mondal T, Naskar S K, Ekbal A, Haque R and Godhavarthy S R 2008 Bengali, Hindi and Telugu to English ad-hoc bilingual task at CLEF 2007. In: Advances in Multilingual and Multimodal Information Retrieval, pp. $88-94$

[9] Chinnakotla M K, Ranadive S, Damani O P and Bhattacharyya P 2008 Hindi to English and Marathi to English cross language information retrieval evaluation. In Advances in Multilingual and Multimodal Information Retrieval, pp. 111-118

[10] Pingali P and Varma V 2007 IIIT hyderabad at CLEF 2007-adhoc Indian language CLIR task. In Working Notes for the CLEF 2007 Workshop

[11] Yu F, Zheng D, Zhao T, Li S and Yu H 2006 Chinese-english cross-lingual information retrieval based on domain ontology knowledge. In: Proceedings of the 2006 IEEE - International Conference on Computational Intelligence and Security, vol. 2, pp. 1460-1463

[12] Yahya Z, Abdullah M T, Azman A and Kadir R A 2013 Query translation using concepts similarity based on quran ontology for cross-language information retrieval. J. Comput. Sci. 9(7): 889-897

[13] Abusalah M, Tait J and Oakes M 2009 Cross language information retrieval using multilingual ontology as translation and query expansion base. Polibits (40): 13-16

[14] Monti J, Monteleone M, Buono M P and Marano F 2013 Natural language processing and big data-an ontologybased approach for cross-lingual information retrieval. In: Proceedings of the 2013 IEEE-International Conference on Social Computing (SocialCom), pp. 725-731

[15] Pourmahmoud S and Shamsfard M 2008 Semantic crosslingual information retrieval. In: Proceedings of the $23 \mathrm{rd}$ IEEE - International Symposium on Computer and Information Sciences, ISCIS'08, pp. 1-4

[16] Navigli R and Ponzetto S P 2012 Babelnet: the automatic construction, evaluation and application of a wide-coverage multilingual semantic network. J. Comput. Sci. 193: 217-250

[17] Nastase V and Strube M 2013 Transforming wikipedia into a large scale multilingual concept network. Artif. Intell. 194: $62-85$

[18] Xu R, Gao Z, Pan Y, Qu Y and Huang Z 2008 An integrated approach for automatic construction of bilingual ChineseEnglish wordnet. In: Proceedings of ASWC 2008: The Semantic Web, pp. 302-314 
[19] Thenmozhi D and Aravindan C 2016 An automatic and clause based approach to learn relations for ontologies. Comput. J. 59(6): 889-907

[20] Bhogal J, Macfarlane A and Smith P 2007 A review of ontology based query expansion. Inf. Process. Manag. 43(4): 866-886

[21] Jain V and Singh M 2013 Ontology based information retrieval in semantic web: a survey. Int. J. Inf. Technol. Comput. Sci. 5(10): 62-69

[22] Sy M F, Ranwez S, Montmain J, Regnault A, Crampes M and Ranwez V 2012 User centered and ontology based information retrieval system for life sciences. BMC Bioinf. 13(Suppl 1): S4

[23] Sujatha P and Dhavachelvan P 2011 A review on the cross and multilingual information retrieval. Int. J. Web Semant. Technol. 2(4): 115-124

[24] Sorg P and Cimiano P 2012 Exploiting wikipedia for crosslingual and multilingual information retrieval. Data Knowl. Eng. 74: 26-45

[25] Majumder P, Mitra M, Parui S K and Bhattacharyya P 2007 Initiative for Indian language IR evaluation. In: Proceedings of the First International Workshop on Evaluating Information Access (EVIA), Tokyo, Japan, May 15
[26] Mandal D, Dandapat S, Gupta M, Banerjee P and Sarkar S 2007 Bengali and Hindi to English cross-language text retrieval under limited resources. In: Working Notes for the CLEF 2007 Workshop

[27] Jagarlamudi J and Kumaran A 2008 Cross-lingual information retrieval system for Indian languages. In: Proceedings of the Advances in Multilingual and Multimodal Information Retrieval Workshop, pp. 80-87

[28] Rao T P R K and Devi S L 2013 Tamil English cross lingual information retrieval. In: Multilingual Information Access in South Asian Languages, pp. 269-279

[29] Thenmozhi D and Aravindan C 2009 Tamil-English cross lingual information retrieval system for agriculture society. In: Proceedings of the Tamil Internet Conference, pp. $173-178$

[30] Popovic M and Ney H 2006 POS-based word reorderings for statistical machine translation. In: Proceedings of the International Conference on Language Resources and Evaluation, pp. 1278-1283

[31] Menon A G, Saravanan S, Loganathan R and Soman K 2009 Amrita morph analyzer and generator for tamil: a rule based approach. In: Proceedings of the Tamil Internet Conference, pp. 239-243 